ISSN 1855-3966 (printed edn.), ISSN 1855-3974 (electronic edn.)

ARS MATHEMATICA CONTEMPORANEA 22 (2022) \#P2.05

https://doi.org/10.26493/1855-3974.1955.1cd

(Also available at http://amc-journal.eu)

\title{
Notes on weak-odd edge colorings of digraphs*
}

\author{
César Hernández-Cruz (D) \\ Facultad de Ciencias, Universidad Nacional Autónoma de México, \\ Av. Universidad 3000, Circuito Exterior S/N, Ciudad Universitaria, CDMX, México \\ Mirko Petruševski ${ }^{\dagger}$ \\ Faculty of Mechanical Engineering - Skopje, University Ss Cyril and Methodius, \\ 1000 Skopje, Macedonia \\ Riste Škrekovski \\ FMF, University of Ljubljana, 1000 Ljubljana, Slovenia, and \\ Faculty of Information Studies, 8000 Novo mesto, Slovenia, and \\ University of Primorska, FAMNIT, 6000 Koper, Slovenia
}

Received 18 March 2019, accepted 29 July 2021, published online 27 May 2022

\begin{abstract}
A weak-odd edge coloring of a general digraph $D$ is a (not necessarily proper) coloring of its edges such that for each vertex $v \in V(D)$ at least one color $c$ satisfies the following conditions: if $d_{D}^{-}(v)>0$ then $c$ appears an odd number of times on the incoming edges at $v$; and if $d_{D}^{+}(v)>0$ then $c$ appears an odd number of times on the outgoing edges at $v$. The minimum number of colors sufficient for a weak-odd edge coloring of $D$ is the weak-odd chromatic index, denoted $\chi_{\text {wo }}^{\prime}(D)$. It is known that $\chi_{\text {wo }}^{\prime}(D) \leq 3$ for every digraph $D$, and that the bound is sharp. In this article we show that the weak-odd chromatic index can be determined in polynomial time. Restricting to edge colorings of $D$ with at most two colors, the minimum number of vertices $v \in V(D)$ for which no color $c$ satisfies the above conditions is the defect of $D$, denoted $\operatorname{def}(D)$. Surprisingly, it turns out that the problem of determining the defect of digraphs is (polynomially) equivalent to the problem of finding the matching number of simple graphs. Moreover, we characterize the classes of associated digraphs and tournaments in terms of the weak-odd chromatic index and the defect.
\end{abstract}

Keywords: Digraph, weak-odd edge coloring, weak-odd chromatic index, defective coloring, tournament.

Math. Subj. Class. (2020): 05C15, 05C20

\footnotetext{
*This work is partially supported by ARRS Program P1-0383 and ARRS Project J1-1692.

$\dagger$ Corresponding author.

E-mail addresses: japo@ ciencias.unam.mx (César Hernández-Cruz), mirko.petrushevski@gmail.com (Mirko Petruševski), skrekovski@gmail.com (Riste Škrekovski)
} 


\section{Introduction}

Throughout the article we mainly follow terminology and notation used in [1]. All graphs and digraphs are considered to be finite. Loops, parallel edges and parallel arcs are admissible, i.e., strictly speaking, we consider the general setup of pseudographs and directed pseudographs, but in order to avoid lengthy terminology, we abbreviate these terms to 'graphs' and 'digraphs', respectively.

Cheilaris et al. [4] introduced the notion of odd coloring of a hypergraph $\mathcal{H}$ as a vertex coloring such that for every (hyper-)edge $e \in \mathcal{E}(\mathcal{H})$ there is a color $c$ with an odd number of vertices of $e$ colored by $c$. Restricting to graphs $G$ (and thus to plain edges), the previous coloring notion is merely the usual notion of 'proper' coloring of $G$. Through interchanging the roles played by vertices and edges, initially motivated by [5, 6], the analogous edge coloring notion for graphs was introduced in [10] as follows. An edge coloring of $G$ is said to be weak-odd if it holds that:

(WO) For every vertex $v \in V(G)$ with degree $d_{G}(v)>0$, at least one color $c$ appears an odd number of times on the set of edges incident with $v$.

The additional adjective 'weak' has been included in the name simply in order to differentiate this from the related, already existing, notion of 'odd edge coloring' of graphs, defined in [12]. (The latter notion has stronger requirements for the colors appearing at a vertex.)

Let us clarify that, by definition, any loop at $v$ colored by $c$ contributes 2 to the count of appearances of $c$ on $E_{G}(v)$ (the set of all edges incident with $v$ ). An obvious necessary and sufficient condition for weak-odd edge colorability of graph $G$ is the absence of 'isolated loops', i.e., nonempty trivial components. A weak-odd edge coloring of $G$ using at most $k$ colors is referred to as a weak-odd $k$-edge coloring of $G$, and such a graph is said to be weak-odd k-edge colorable. The weak-odd chromatic index, $\chi_{\mathrm{wo}}^{\prime}(G)$, is the minimum $k$ for which $G$ is weak-odd $k$-edge colorable. Obviously, apart from 'isolated loops', any other loop addition or removal does not influence the existence nor alters the value of $\chi_{\text {wo }}^{\prime}(G)$. The following characterization of graphs $G$ in terms of $\chi_{\text {wo }}^{\prime}(G)$ was obtained in [10]. It makes use of the next two notions. A graph $G$ is even (resp. odd) if every vertex $v \in V(G)$ has even (resp. odd) degree $d_{G}(x)$.

Theorem 1.1. For any connected graph $G$ whose edge set does not consist entirely of loops, it holds that

$$
\chi_{\mathrm{wo}}^{\prime}(G)= \begin{cases}0 & \text { if } G=K_{1}, \\ 1 & \text { if } G \text { is odd, } \\ 2 & \text { if } G \text { has even order or is not even, } \\ 3 & \text { if } G \text { is even, has odd order, and is not } K_{1} .\end{cases}
$$

This paper treats the analogous coloring notion for digraphs. In the next section we further explain the motivation behind the definition of 'weak-odd edge coloring' of digraphs, introduced in [11], and then show that the corresponding coloring index $\chi_{\text {wo }}^{\prime}$ can be determined in linear time. We also address a related problem concerning the minimum number of vertices at which an arbitrary 2-edge coloring of a digraph fails at being 'weak-odd', and prove a connection with the problem of determining the matching number of a simple graph. In Section 3, the discussion restricts to two common classes of digraphs, namely, 
the class of associated digraphs ${ }^{1}$ and the class of tournaments. For each of these classes we give a descriptive characterization of their members in terms of $\chi_{\text {wo }}^{\prime}$. In the final section we briefly convey our thoughts on possible further work on the topic of weak-odd edge colorings of digraphs. For the end of this introductory section, we mention some common notions and facts that will be frequently used throughout.

\subsection{General terminology and notation}

We denote the symmetric difference of sets $A, B$ by $A \oplus B$. The same notation is in use for the symmetric difference of two spanning subgraphs $A, B$ of a ground graph. Given a graph $G$ and an even-sized subset $T$ of $V(G)$, a spanning subgraph $H$ is a $T$-join of $G$ if $d_{H}(v)$, the degree of $v$ with respect to $H$, is odd for all $v \in T$ and even for all $v \in V(G) \backslash T$. The symmetric difference of a $T^{\prime}$-join and a $T^{\prime \prime}$-join of $G$ is a $T^{\prime} \oplus T^{\prime \prime}$-join, which yields the following classical result (see [14]): every connected graph $G$ contains a $T$-join. In particular, every even-ordered connected graph $G$ has an odd factor (i.e., a $V(G)$-join).

An edge coloring of a graph $G$ (resp. digraph $D$ ) with color set $S$ is an assignment $E(G) \rightarrow S$ (resp. $A(D) \rightarrow S$ ). Every $T$-join of $G$ can be interpreted as an edge coloring with color set $\{1,2\}$ such that 1 is used an odd number of times at each $v \in T$ and and even number of times (possibly zero) at each $v \in V(G) \backslash T$.

Given a digraph $D$ and a vertex $v \in V(D)$, the size of the set $\partial_{D}^{-}(v)$, of incoming edges at $v$, (resp. $\partial_{D}^{+}(v)$, of outgoing edges at $v$,) is the in-degree $d_{D}^{-}(v)$ (resp. out-degree $\left.d_{D}^{+}(v)\right)$ of the vertex $v$; we call each of $\partial_{D}^{-}(v)$ and $\partial_{D}^{+}(v)\left(\operatorname{resp} . d_{D}^{-}(v)\right.$ and $\left.d_{D}^{+}(v)\right)$ a semicut (resp. semi-degree) of $v$. Since loops are allowed, let us clarify that $\partial_{D}^{-}(v) \cap \partial_{D}^{+}(v)$ constitutes the set of loops at $v$; in other words, any loop at vertex $v$ contributes 1 to each semi-degree of $v$, i.e., strictly speaking $d_{D}^{-}(v)$ and $d_{D}^{-}(v)$ are the semi-pseudodegrees of $v$ (the in-pseudodegree and out-pseudodegree, respectively). The sum $d_{D}(v)=d_{D}^{-}(v)+$ $d_{D}^{+}(v)$ is the degree of $v$; a vertex of degree 0 is said to be isolated. Given a nonisolated vertex $v$, if $d_{D}^{-}(v)=0\left(\right.$ resp. $\left.d_{D}^{+}(v)=0\right)$, then $v$ is a source (resp. sink) of $D$. Any source or sink is a peripheral vertex of $D$, whereas a nonisolated vertex that is neither a source nor a sink is an intermediate vertex. A vertex $u$ is said to dominate a vertex $v$ if $v \in \partial_{D}^{+}(u)$; equivalently, $v$ is dominated by $u$.

Two graphs or digraphs are considered identical if they are isomorphic to each other. The numbers of vertices and edges of graph or digraph $D$ are denoted by $n(D)$ and $m(D)$; these basic parameters are the order and size of $D$, respectively; a graph or digraph of order 1 (resp. size 0) is trivial (resp. empty).

A graph is bipartite if its vertex set can be partitioned into two subsets $X$ and $Y$ so that every edge has one end in $X$ and one end in $Y$; such a partition $(X, Y)$ is called a bipartition of the graph, and $X$ and $Y$ its partite sets. We denote a bipartite graph $G$ with bipartition $(X, Y)$ by $G[X, Y]$. Given a digraph $D$, its split (or bipartite representation), $\operatorname{BG}(D)$, is the bipartite graph $G$ whose partite sets $V^{+}, V^{-}$are copies of $V(D)$. For each $v \in V(D)$, there is one vertex $v^{+} \in V^{+}$and one $v^{-} \in V^{-}$. For each directed $u v$-edge in $D$, there is an edge with endpoints $u^{+}$and $v^{-}$in $G$. Hence, the degrees of the vertices $v^{+}, v^{-}$in the split of $D$ are precisely the out-degree and in-degree of $v$ in $D$, respectively; the pair $\left(v^{+}, v^{-}\right)$is obtained by splitting $v$ in regard to $D$. The re-identification of each such pair $\left(v^{+}, v^{-}\right)$into $v$ results in the so-called underlying graph of $D$, denoted $G(D)$.

\footnotetext{
${ }^{1}$ A digraph $D$ is associated if for every $\operatorname{arc}(u, v)$ of $D$, the $\operatorname{arc}(v, u)$ is also present in $D$, and the number of loops at any vertex is even.
} 
Furthermore, any balanced bipartite graph $G$ is a split of some digraph $D$, i.e., can be 'transformed' into $D$ by reversing the described procedure.

The other way around, any graph $G$ can be regarded as a digraph $D(G)$, by replacing each of its edges by two oppositely oriented arcs with the same ends (each loop of $G$ gives rise to two directed loops on the same vertex); this digraph is the associated digraph of $G$. One may also obtain a digraph $D$ from a graph $G$ by replacing each edge by one arc on the same endpoints; such a digraph $D$ is an orientation of $G$. A tournament is an orientation of a complete graph. Conversely, the underlying graph $G(D)$ of a digraph $D$ is obtained by 'forgetting orientation'. A directed path or directed cycle is an orientation of a path or cycle in which each vertex dominates its successor in the sequence.

A digraph $D$ is said to be strongly connected (or simply strong) if for any pair $u, v$ of its vertices there is a directed $u v$-path, i.e., a directed path joining vertex $u$ with vertex $v$. Given a digraph $D$, every maximal strong subdigraph of $D$ is a strong component of $D$. The condensation $C(D)$ of a digraph $D$ is the loopless directed (multi)graph whose vertices correspond to the strong components of $D$, with any two vertices of $C(D)$ being linked by as many directed edges as there are directed edges in $D$ linking the corresponding strong components, and with the consistent orientation. The peripheral strong components of $D$ which correspond to the vertices of $C(D)$ that are sources (resp. sinks), are the initial (resp. terminal) strong components; the remaining strong components of $D$ are called intermediate or isolated according to the nature of the corresponding vertices in $C(D)$.

\section{Weak-odd edge colorings of digraphs}

In the realm of digraphs $D$, when defining the notion of 'weak-odd edge coloring' two options come to mind. Initially, one may obtain a 'directed version' of the condition (WO) as follows:

$(\overrightarrow{\mathrm{W}} \overrightarrow{\mathrm{O}})$ For every vertex $v \in V(D)$, on each nonempty semi-cut of $v$ at least one color $c$ appears an odd number of times.

However, the above 'definition' fails to capture the essence of digraphs since it basically ignores the fact that arc sets $\partial_{D}^{-}(v)$ and $\partial_{D}^{+}(v)$ are incident with a common vertex (namely, $v$ ). Actually, a moment's thought reveals that, if we decide to adopt the initial definition, then this coloring notion for digraphs would be merely a 'disguise' of weak-odd edge coloring of bipartite graphs with equally sized partite sets. Namely, it can readily be seen that an edge coloring of $D$ satisfies condition $(\overrightarrow{\mathrm{W}} \overrightarrow{\mathrm{O}})$ if and only if the induced edge coloring on $\mathrm{BG}(D)$ satisfies condition (WO). Consequently, in view of Theorem 1.1, every digraph $D$ admits a 3-edge coloring obeying $(\overrightarrow{\mathrm{W}} \overrightarrow{\mathrm{O}})$; moreover, three colors are indeed required if and only if at least one component of $\mathrm{BG}(D)$ is a nonempty even graph of odd order. One such example is depicted in Figure 1. Notice that if at least one of the loops is removed from $D$ then the obtained digraph would admit a 2-edge coloring that satisfies condition $(\overrightarrow{\mathrm{W}} \overrightarrow{\mathrm{O}})$.

As noticed, unlike for graphs, in the realm of digraphs the presence of loops may influence the value of the corresponding chromatic index in a nontrivial manner. This is so because any such loop in the digraph is no longer a loop in its split.

A more appropriate definition of the notion of 'weak-odd edge coloring' for digraphs, the one we shall adopt in this study, is obtained as follows. Recall that any graph $G$ can be seen as a digraph, the associated digraph $D(G)$. In the obvious fashion, every edge 

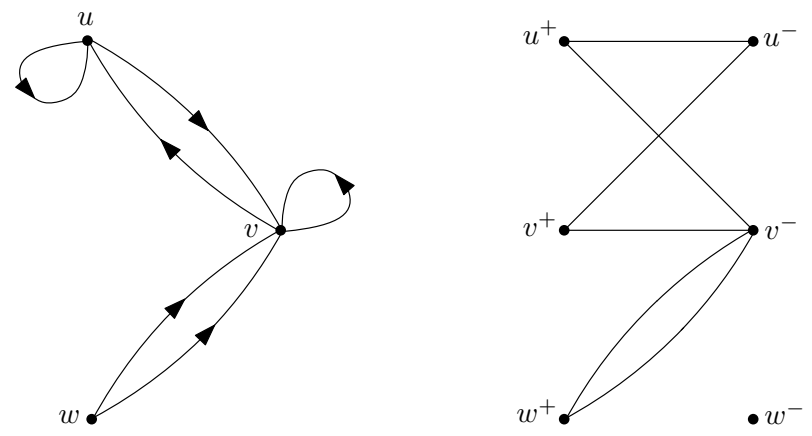

Figure 1: (left) A digraph $D$ that fails condition $(\overrightarrow{\mathrm{W}} \overrightarrow{\mathrm{O}})$ under any 2-edge coloring, and (right) its split $\mathrm{BG}(D)$ with the left-hand (resp. right-hand) partite set representing $V^{+}$ (resp. $V^{-}$). The nonempty component of $\mathrm{BG}(D)$ is an even graph of odd order.
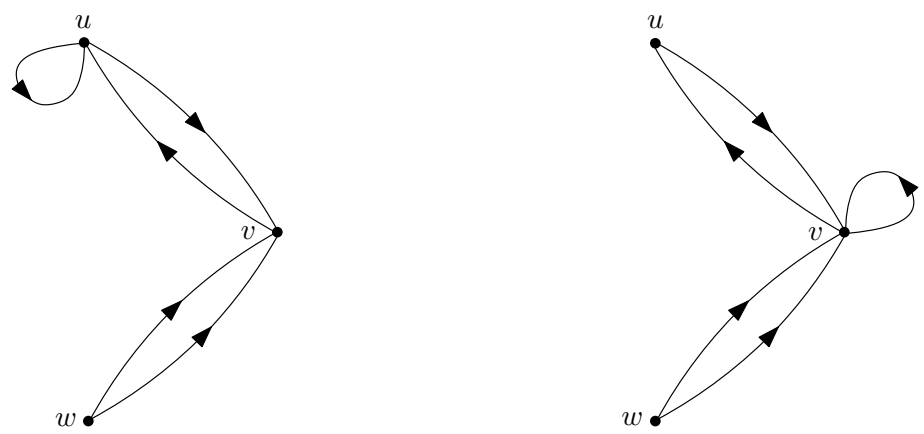

Figure 2: Digraphs $D_{1}$ (left) and $D_{2}$ (right) are obtained from the digraph in Figure 1 by removing a certain loop (at $v$ and at $u$, respectively). Both admit 2-edge colorings fulfilling condition $(\overrightarrow{\mathrm{W}} \overrightarrow{\mathrm{O}})$, but none admits a 2-edge coloring satisfying $(\overrightarrow{\mathrm{WO}})$.

coloring $\varphi$ of $G$ can be interpreted as an edge coloring $\varphi_{D}$ of $D(G)$. Notice that if $\varphi$ is weak-odd then $\varphi_{D}$ satisfies the condition $(\overrightarrow{\mathrm{WO}})$ below, which states a stronger requirement than the one imposed by $(\overrightarrow{\mathrm{W}} \overrightarrow{\mathrm{O}})$. This particular reasoning served as the motivation in [11] for defining an edge coloring of a digraph $D$ to be weak-odd if:

$(\overrightarrow{\mathrm{WO}})$ For every vertex $v \in V(D)$, at least one color $c$ appears an odd number of times on each nonempty semi-cut of $v$.

Same as with $(\overrightarrow{\mathrm{W}} \overrightarrow{\mathrm{O}})$, in case $v$ is a sink (resp. source), the condition $(\overrightarrow{\mathrm{WO}})$ amounts to the appearance of $c$ an odd number of times on the incut $\partial_{D}^{-}(v)$ (resp. outcut $\partial_{D}^{+}(v)$ ). The difference between $(\overrightarrow{\mathrm{W}} \overrightarrow{\mathrm{O}})$ and $(\overrightarrow{\mathrm{WO}})$ is reflected at the intermediate vertices (cf. Figure 2).

The minimum number of colors sufficient for a weak-odd edge coloring of a digraph $D$ is the weak-odd chromatic index, denoted $\chi_{\text {wo }}^{\prime}(D)$. A weak-odd edge coloring of $D$ using at most $k$ colors is referred to as a weak-odd $k$-edge coloring, and any such $D$ is said to be weak-odd k-edge colorable. Hence, $\chi_{\text {wo }}^{\prime}(D)$ is the minimum $k$ for which $D$ is weak-odd $k$-edge colorable. 
Interestingly, analogous to graphs, the same upper bound (of three colors) holds for the weak-odd chromatic index of digraphs. Namely, the following was proven in [11].

Theorem 2.1. Every digraph is weak-odd 3-edge colorable.

As already illustrated through Figures 1 and 2, the bound $\chi_{\text {wo }}^{\prime}(D) \leq 3$ is sharp, i.e., not every digraph is weak-odd 2 -edge colorable. Analogous to the setting of graphs, it is quite trivial to characterize which digraphs are weak-odd 1-edge colorable. Indeed, the inequality $\chi_{\mathrm{wo}}^{\prime}(D) \leq 1$ holds if and only if for every vertex $v \in V(D)$ both semi-degrees $d_{D}^{-}(v), d_{D}^{+}(v)$ are odd or zero. Furthermore, $\chi_{\text {wo }}^{\prime}(D)=0$ holds precisely when $D$ is empty. Thus, in order to characterize all digraphs in terms of their weak-odd chromatic index, it suffices to figure out which are the weak-odd 2-edge colorable ones.

\subsection{Characterization of weak-odd 2-edge colorability}

The partial split, $\mathrm{PS}(D)$, of given digraph $D$ is a graph obtained by splitting (in regard to $D$ ) only those vertices $v \in V(D)$ for which at least one semi-degree is even (including zero), and then forgetting orientation. In other words, $\operatorname{PS}(D)$ is the graph obtained from $\mathrm{BG}(D)$ by re-identifying each pair $\left(u^{+}, u^{-}\right)$for which both $d_{D}^{+}(u)$ and $d_{D}^{-}(u)$ are odd (cf. Figure 3). In particular, if no vertex of $D$ has only odd-sized semi-cuts, then $\operatorname{PS}(D)$ is the same graph as the split $\mathrm{BG}(D)$; contrarily, if every nonisolated vertex of $D$ has only odd-sized semi-cuts, then $\operatorname{PS}(D)$ is the same as the underlying graph $G(D)$. However, in general, these three graphs related to $D$ differ from each other.
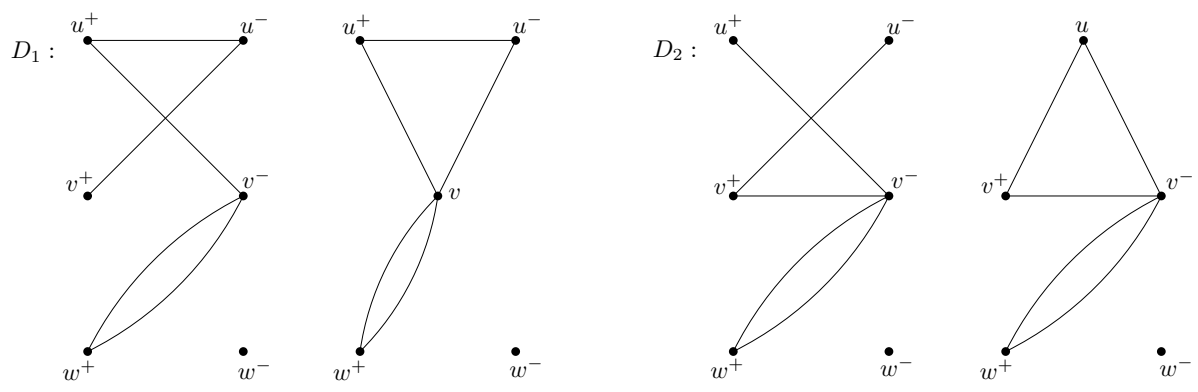

Figure 3: The split $\mathrm{BG}\left(D_{1}\right)$ and partial split $\operatorname{PS}\left(D_{1}\right)$ (left), and the split $\mathrm{BG}\left(D_{2}\right)$ and partial split $\operatorname{PS}\left(D_{2}\right)$ (right), where $D_{1}, D_{2}$ are the digraphs from Figure 2. The induced 3-partition $\left\{V_{1}, V_{2}, V_{3}\right\}$ of $V\left(\operatorname{PS}\left(D_{1}\right)\right)$ consists of $V_{1}=\{v\}, V_{2}=\left\{u^{+}, u^{-}, w^{+}, w^{-}\right\}$ and $V_{3}=\varnothing$, whereas the corresponding 3-partition of $V\left(\operatorname{PS}\left(D_{2}\right)\right)$ has $V_{1}=\{u\}$, $V_{2}=\left\{v^{+}, v^{-}, w^{+}, w^{-}\right\}, V_{3}=\varnothing$.

We distinguish between three types of vertices in $\operatorname{PS}(D)$ inducing a 3-partition $\left\{V_{1}, V_{2}, V_{3}\right\}$ of $V(\mathrm{PS}(D))$ :

- the first type of vertices, constituting $V_{1}$, are the members of $V(D) \cap V(\operatorname{PS}(D))$, i.e., the vertices $u$ of $D$ having odd semi-degrees $d_{D}^{+}(u), d_{D}^{-}(u)$.

- the second type of vertices, forming $V_{2}$, are the members $v$ of $V(\operatorname{PS}(D)) \backslash V(D)$ that have even degree $d_{\mathrm{PS}(D)}(v)$.

- finally, the third type of vertices, comprising $V_{3}$, are the members $w$ of $V(\operatorname{PS}(D)) \backslash$ $V(D)$ that have odd degree $d_{\mathrm{PS}(D)}(w)$. 
For simplicity of presentation, on several occasions we shall employ the following adhoc terminology and notation. Given a graph $G$, a vertex $x \in V(G)$ is said to be even (resp. odd) if $d_{G}(x)$ is even (resp. odd). The set of even (resp. odd) vertices of $G$ is denoted $\operatorname{Even} V(G)$ (resp. $O d d V(G)$ ). Note that, by the handshake lemma, $O d d V(G)$ is always even-sized. Thus, $V_{3}$ above equals $O d d V(\operatorname{PS}(D))$, whereas $V_{1} \bullet V_{2}=\operatorname{Even} V(\operatorname{PS}(D))$.

Theorem 2.2. A digraph $D$ is weak-odd 2-edge colorable if and only if for every nonempty component $K$ of $\operatorname{PS}(D)$ we have that $V(K) \cap V_{2}$ is even-sized or $V(K) \cap V_{3} \neq \varnothing$.

Proof. Assuming $\chi_{\text {wo }}^{\prime}(D) \leq 2$, let $\varphi: A(D) \rightarrow\{1,2\}$ be a weak-odd edge coloring of $D$ and consider the induced edge coloring of $\operatorname{PS}(D)$. Observe that for every vertex $u \in V_{1}$, each of the colors 1 and 2 is used an even number of times on $E_{\mathrm{PS}(D)}(u)$. Indeed, the edge set $E_{\mathrm{PS}(D)}(u)$ corresponds to the entire cut $\partial_{D}(u)=\partial_{D}^{+}(u) \bullet \partial_{D}^{-}(u)$; thus, since both constituents in this disjoint union are odd-sized, for every color $c \in\{1,2\}$ the parities of $\left|\partial_{D}^{+}(u) \cap \varphi^{-1}(c)\right|$ and $\left|\partial_{D}^{-}(u) \cap \varphi^{-1}(c)\right|$ are equal. In contrast, for every nonisolated vertex $v \in V_{2}$, each of the colors 1 and 2 appears an odd number of times on $E_{\mathrm{PS}(D)}(v)$. The reason behind this is that the edge set $E_{\mathrm{PS}(D)}(v)$ corresponds to a nonempty even-sized semi-cut of a vertex in $D$.

Therefore, if $K$ is a nonempty component of $\operatorname{PS}(D)$ such that $V(K) \subseteq V_{1} \bullet V_{2}$, then each of the two color classes induces in $K$ a subgraph $K_{i}(i \in\{1,2\})$ such that $\operatorname{Odd} V\left(K_{i}\right)=V(K) \cap V_{2}$. Consequently, the intersection $V(K) \cap V_{2}$ is even-sized.

Arguing in the opposite direction, assume now that every nonempty component $K$ of $\operatorname{PS}(D)$ meets the stated requirements. We construct an assignment $E(K) \rightarrow\{1,2\}$ as follows. If $V(K) \cap V_{2}$ is even-sized, then define $T=V(K) \cap V_{2}$. Otherwise, select an odd-sized subset $S_{K} \subseteq V(K) \cap V_{3}$ and define $T=\left(V(K) \cap V_{2}\right) \cup S_{K}$. Either way, $T$ is an even-sized subset of $V(K)$. Therefore, there exists a $T$-join $H$ of $K$. Color $E(H)$ with 1 and $E(K) \backslash E(H)$ with 2 . Consider the induced edge coloring of $D$, and observe the following.

(1) At each vertex $u \in V(D) \cap V_{1}$, precisely one of the colors 1,2 satisfies condition $(\overrightarrow{\mathrm{WO}})$. Indeed, by construction, each color is used an even number of times on $\partial_{D}(u)$, and thus has equal parities of appearance on the odd-sized sets $\partial_{D}^{-}(u)$ and $\partial_{D}^{+}(u)$, respectively.

(2) At each nonisolated vertex $v \in V(D) \backslash V_{1}$ such that the vertices $v^{+}, v^{-} \in V_{2}$, colors 1 and 2 both satisfy condition $(\overrightarrow{\mathrm{WO}})$. Namely, by construction, color 1 is used an odd number of times on each nonempty semi-cut of $v$; on the other hand, both $\partial_{D}^{-}(v)$ and $\partial_{D}^{+}(v)$ are even-sized.

(3) At each vertex $w \in V(D) \backslash V_{1}$ such that one of the vertices $w^{+}, w^{-}$belongs in $V_{2}$ (and the other in $\left.V_{3}\right)$, precisely one of the colors 1,2 satisfies condition $(\overrightarrow{\mathrm{WO}})$. Indeed, if the 'half' of $w$ belonging to $V_{3}$ is used in some $S_{K}$ then color 1 meets $(\overrightarrow{\mathrm{WO}})$; otherwise, color 2 does so.

Thus, the digraph $D$ is weak-odd 2-edge colorable.

For example, the digraph $D$ depicted in Figure 4 is weak-odd 2-edge colorable because the only nonempty component of $\operatorname{PS}(D)$ intersects $V_{3}$. With the notation employed in the 
proof, if we take $E(H)$ to consist of $x^{+} y^{-}, y^{+} u$, and a $u z^{-}$edge, then the induced weakodd 2-edge coloring of $D$ assigns color 1 to $x y, y u$ and a $u z$ arc, and assigns color 2 to the rest of $A(D)$.
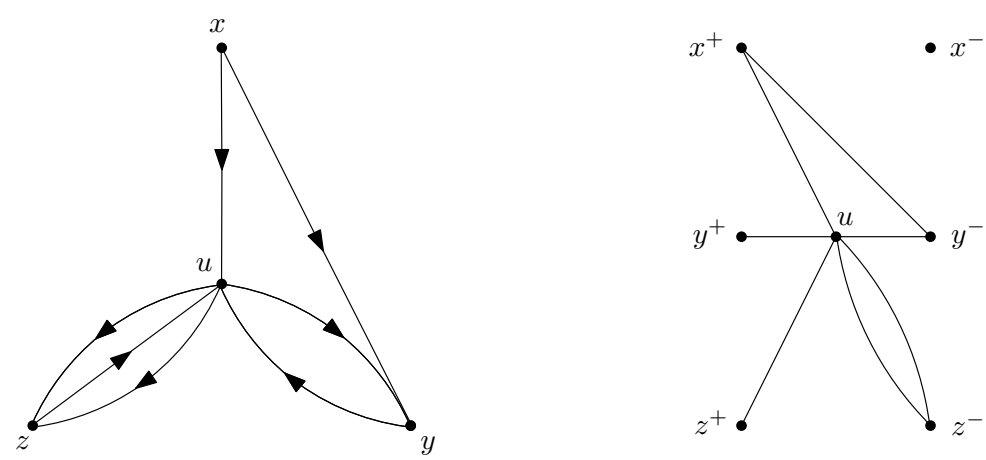

Figure 4: A digraph $D$ (left) and its partial split $\operatorname{PS}(D)$ (right). The induced 3-partition has $V_{1}=\{u\}, V_{2}=\left\{x^{+}, x^{-}, y^{-}, z^{-}\right\}$and $V_{3}=\left\{y^{+}, z^{+}\right\}$.

Contrarily, the digraph $D$ depicted in Figure 5 is not weak-odd 2-edge colorable, since the induced 3-partition has $V_{1}=\{u\}, V_{2}=\left\{x^{+}, x^{-}, y^{+}, y^{-}, z^{+}, z^{-}\right\}$and $V_{3}=\varnothing$, the only nonempty component of its partial split does not intersect $V_{3}$ and contains an odd number of vertices from $V_{2}$.
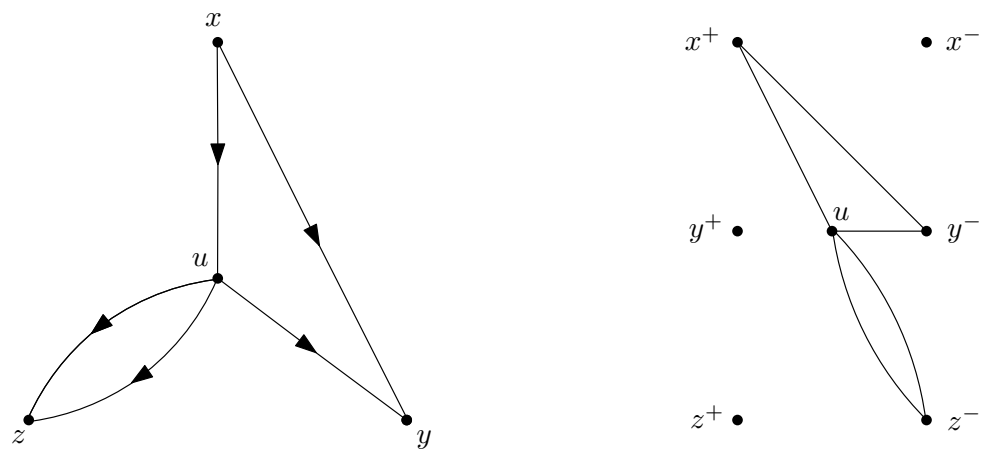

Figure 5: A digraph $D$ (left) and its partial split $\operatorname{PS}(D)$ (right).

The proof of Theorem 2.2 and the fact that the problem of constructing a $T$-join of any connected graph $G$ for a given even-sized subset $T$ of $V(G)$ is solvable in linear time (see [14]), imply that the decision problem of whether $\chi_{\text {wo }}^{\prime}(D) \leq 2$ is solvable in linear time; in the affirmative case, a weak-odd 2-edge coloring of $D$ can be found in linear time. Thus, in view of Theorem 2.1 and the subsequent discussion, we conclude the following.

Corollary 2.3. The weak-odd chromatic index of any digraph $D$ and a corresponding weak-odd $\chi_{\mathrm{wo}}^{\prime}(D)$-edge coloring can be determined in linear time.

To end this subsection we point out an infinite family of digraphs having weak-odd chromatic index equal to 3. A digraph is said to be even if every vertex $v \in V(D)$ is of 
even degree $d_{D}(v)$; in other words, the requirement is that the semi-degrees of $v$ are of equal parity.
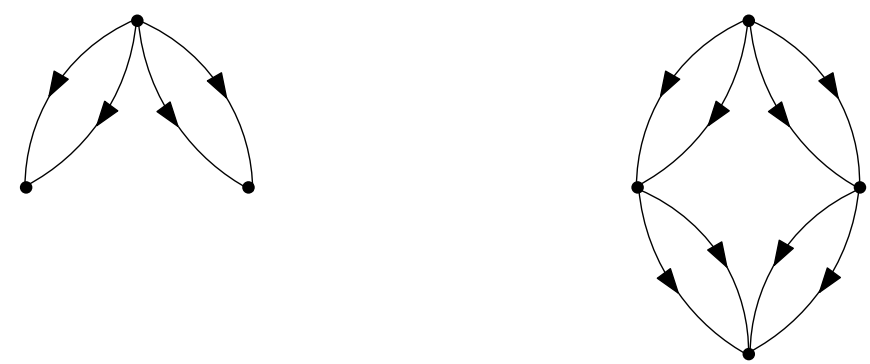

Figure 6: Two even digraphs with weak-odd chromatic index 3.

Proposition 2.4. If an even digraph $D$ has an odd number of peripheral vertices, then $\chi_{\text {wo }}^{\prime}(D)=3$.

Proof. We may assume that $D$ is connected. Consider the partial split $\operatorname{PS}(D)$ of $D$ and the induced 3-partition $\left\{V_{1}, V_{2}, V_{3}\right\}$. Obviously, $V_{3}=\varnothing$ and the number of isolated vertices of $\operatorname{PS}(D)$ equals the number of peripheral vertices of $D$. However, this implies that the number of nonisolated vertices of $\operatorname{PS}(D)$ which belong in $V_{2}$ is odd. Therefore, an odd number of nonempty components of $\operatorname{PS}(D)$ fail to meet the requirements of Theorem 2.2.

Notice that out of the two even digraphs depicted in Figure 6, only the left one has an odd number of peripheral vertices; nevertheless, neither is weak-odd 2-edge colorable. This demonstrates that the condition 'an odd number of peripheral vertices' from the statement of Proposition 2.4, although sufficient, is by no means necessary.

\subsection{Defective weak-odd 2-edge colorings}

The following straightforward result serves as our motivation for the brief discussion within this subsection. In a way, it tells that every graph can be almost weak-odd 2-edge colored.

Proposition 2.5. Every connected graph $G$ admits a 2-edge coloring such that condition (WO) is satisfied at each vertex apart from a prescribed vertex $v \in V(G)$.

Proof. We construct an even-sized subset $T$ of $V(G)$ as follows. If $n(G)$ is even, then define $T=V(G)$; otherwise, let $T=V(G) \backslash\{v\}$. Since $G$ is connected, consider a $T$-join $H$ of $G$. Color $E(H)$ with 1 , and the rest of $E(G)$ with 2 . The obtained 2-edge coloring of $G$ clearly fulfills condition (WO) at each vertex differing from $v$.

One naturally wonders if there exists an analogous result for digraphs that bounds (presumably with 1 ) the number of 'defective vertices', in regard to condition $(\overrightarrow{\mathrm{WO}})$, for some 2-edge coloring? Unfortunately, in contrast to graphs, there are connected digraphs such that for any 2-edge coloring the condition $(\overrightarrow{\mathrm{WO}})$ fails at an unbounded number of vertices.

To construct examples, consider as a 'gadget digraph' $D$ the right-hand digraph from Figure 6 . Observe that no 2 -edge coloring of $D$ can fulfill condition $(\overrightarrow{\mathrm{WO}})$ at all vertices 
excepting the sink (or the source). Thus, by taking any number, say $n$, of copies of $D$ and identifying their sinks (cf. Figure 7) we obtain a connected digraph $D_{n}^{\prime}$ such that under any 2-edge coloring at least $n$ of its vertices are 'defective' in regard to condition $(\overrightarrow{\mathrm{WO}})$. A similar construction using the same gadget graph shows that even strong connectedness comes to no avail in this regard. Namely, take an arbitrary number $n \geq 2$ of copies of $D$, arrange them in circular order and then identify pairwise the corresponding sink and source of each neighboring copies (cf. Figure 7). Once again, the obtained strong digraph $D_{n}^{\prime \prime}$ under any 2-edge coloring has at least $n$ 'defective' vertices in regard to condition $(\overrightarrow{\mathrm{WO}})$. The following question comes to mind: Does anything change in regard to this problem if we confine to simple digraphs, or even more so, to digraphs with simple underlying graphs? The next result answers the question in negative.
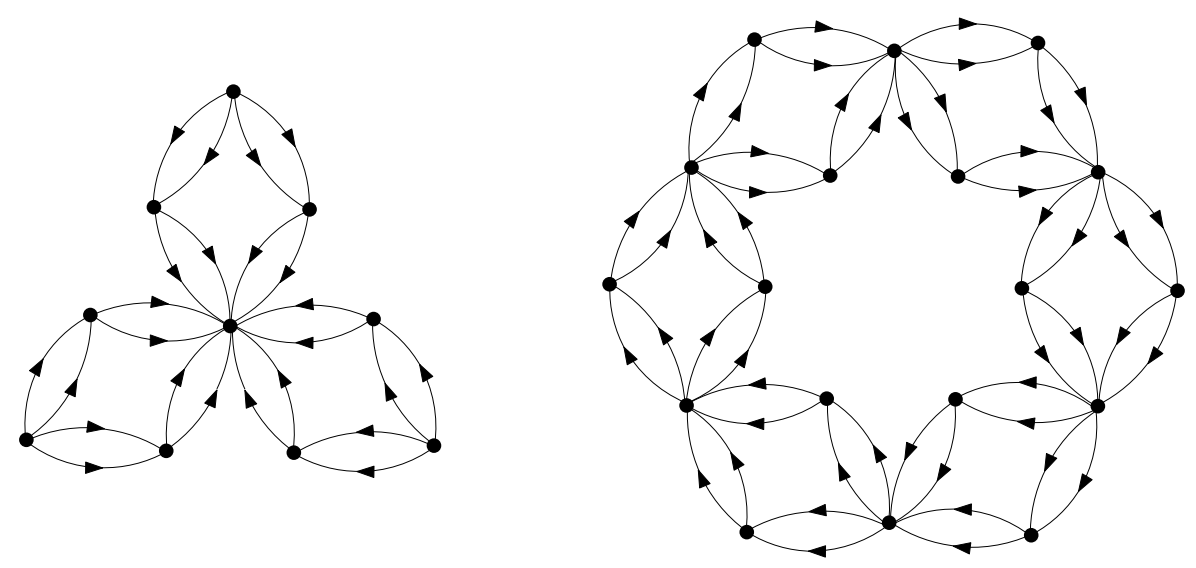

Figure 7: The digraph $D_{3}^{\prime}$ (left), and the digraph $D_{6}^{\prime \prime}$ (right).

Proposition 2.6. For any given positive integer n, there exists a strongly connected digraph $D$ with simple underlying graph $G(D)$ such that under any 2-edge coloring of $D$ at least $n$ of its vertices are 'defective' in regard to condition $(\overrightarrow{\mathrm{WO}})$.

Proof. Simply take $D$ to be a complete subdivision of $D_{n}^{\prime \prime}$. In other words, subdivide (at least once) each arc $e \in A\left(D_{n}^{\prime \prime}\right)$ and orient the newly formed arcs consistently with $e$.

Given a digraph $D$, let $\operatorname{def}(D)$, the defect of $D$, denote the minimum number of 'defective vertices' in regard to condition $(\overrightarrow{\mathrm{WO}})$ taken over all 2-edge colorings of $D$. A question that naturally arises is whether this parameter can be effectively determined. As it turns out, the parameter $\operatorname{def}(D)$ is closely related to yet another graph construction, relating a simple graph $G_{D}$ to each digraph $D$, which we describe next.

Start from the induced subgraph $\mathrm{BC}(D) \subseteq \mathrm{PS}(D)$ that consists of the 'bad components' of $\operatorname{PS}(D)$ in regard to the requirement of Theorem 2.2; in other words, $V(\mathrm{BC}(D))=\cup_{K} V(K)$, where the union is taken over all components $K$ of $\operatorname{PS}(D)$ such that $V(K) \cap V_{2}$ is odd-sized and $V(K) \cap V_{3}$ is empty.

Thus, the vertex set of $G_{D}$ consists of vertices $v_{K}$ corresponding to components $K$ of $\mathrm{BC}(D)$. As for the edge set of $G_{D}$, make two distinct vertices $v_{K^{\prime}}$ and $v_{K^{\prime \prime}}$ adjacent if the respective bad components $K^{\prime}$ and $K^{\prime \prime}$ contain the 'halves' $v^{+}$and $v^{-}$of some vertex 
$v \in V(D)$. To exemplify, we shall make use of the digraphs $D_{n}^{\prime}$ and $D_{n}^{\prime \prime}$ defined above. For the first of these digraphs, it is readily seen that each of the $n+1$ nonempty components of $\operatorname{PS}\left(D_{n}^{\prime}\right)$ is bad, and that $G_{D_{n}^{\prime}}=K_{1, n}$ (cf. Figure 8).
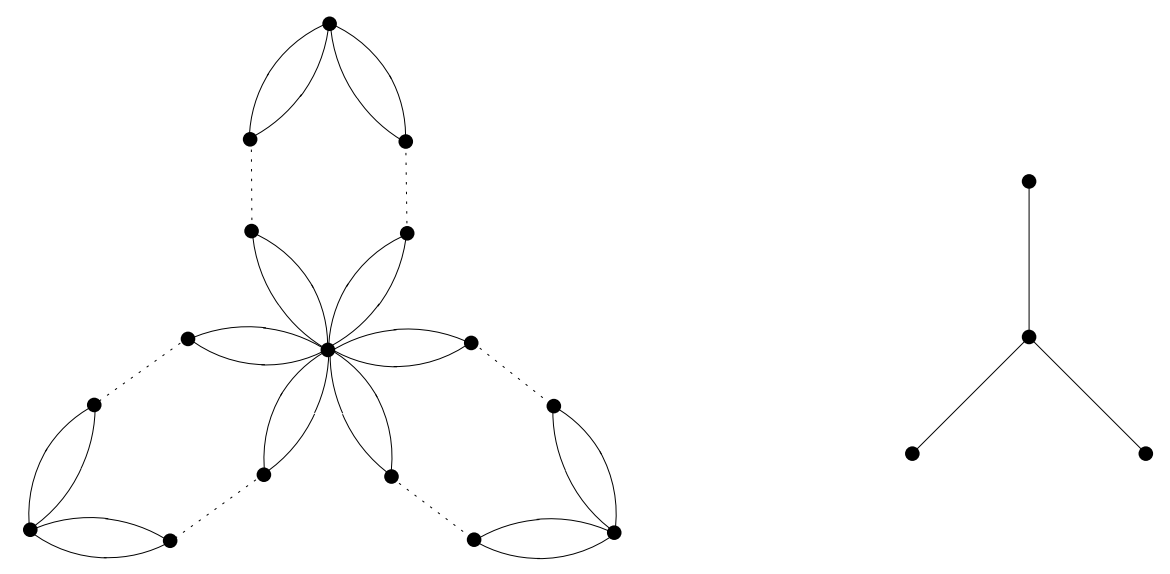

Figure 8: The graph $\mathrm{BC}\left(D_{3}^{\prime}\right)$ (left) with each dotted line matching the two 'halves' of a splitted vertex of $D_{3}^{\prime}$, and the graph $G_{D_{3}^{\prime}}=K_{1,3}$ (right).

Similarly, each of the $2 n$ nonempty components of $\operatorname{PS}\left(D_{n}^{\prime \prime}\right)$ is bad; this time it holds that $G_{D_{n}^{\prime \prime}}=C_{2 n}$ (cf. Figure 9).

Interestingly, any simple graph $G$ is a realization of some $G_{D}$.

Proposition 2.7. For any simple graph $G$ there exists a digraph $D$ such that $G_{D}=G$.

Proof. Let $n=n(G)$ and $m=m(G)$ be the order and size of $G$, respectively. An open $2 m$-necklace is a digraph obtained as follows: take a path $P$ of length $2 m$, replace each edge $e \in E(P)$ by a pair of parallel edges, and then orient each such pair consistently so that with any natural ordering the vertices become: sink, source, sink, ..., source, sink (cf. Figure 10).

Take $n$ disjoint open $2 m$-necklaces, and enumerate them as $D_{1}, \ldots, D_{n}$. Consider an enumeration of the set $E(G)=\left\{e_{1}, \ldots, e_{m}\right\}$. For each $i \in\{1, \ldots, n\}$, fix a natural ordering of the vertices of $D_{i}$, and enumerate them accordingly as $e_{0, i}, e_{1, i}^{+}, e_{1, i}^{-}, \ldots, e_{m, i}^{+}, e_{m, i}^{-}$.

Let $D$ be the digraph obtained from $D_{1} \bullet \cdots \bullet D_{n}$ as follows. Take an enumeration of the vertex set $V(G)=\left\{v_{1}, \ldots, v_{n}\right\}$. For each $e_{k} \in E(G)$, if $e_{k}=v_{i} v_{j}$ with $i<j$, then identify $e_{k, i}^{+}$with $e_{k, j}^{-}$. Observe that, by construction:

- $\operatorname{PS}(D)$ has $n$ nonempty components;

- each such component belongs to $\mathrm{BC}(D)$;

- $G_{D}=G$.

The last item concludes our proof.

Recall that a matching in a graph is a set of pairwise nonadjacent edges that are not loops. If $M$ is a matching, the two ends of each edge of $M$ are said to be matched under $M$, and each vertex incident with an edge of $M$ is said to be covered by $M$. A maximum 

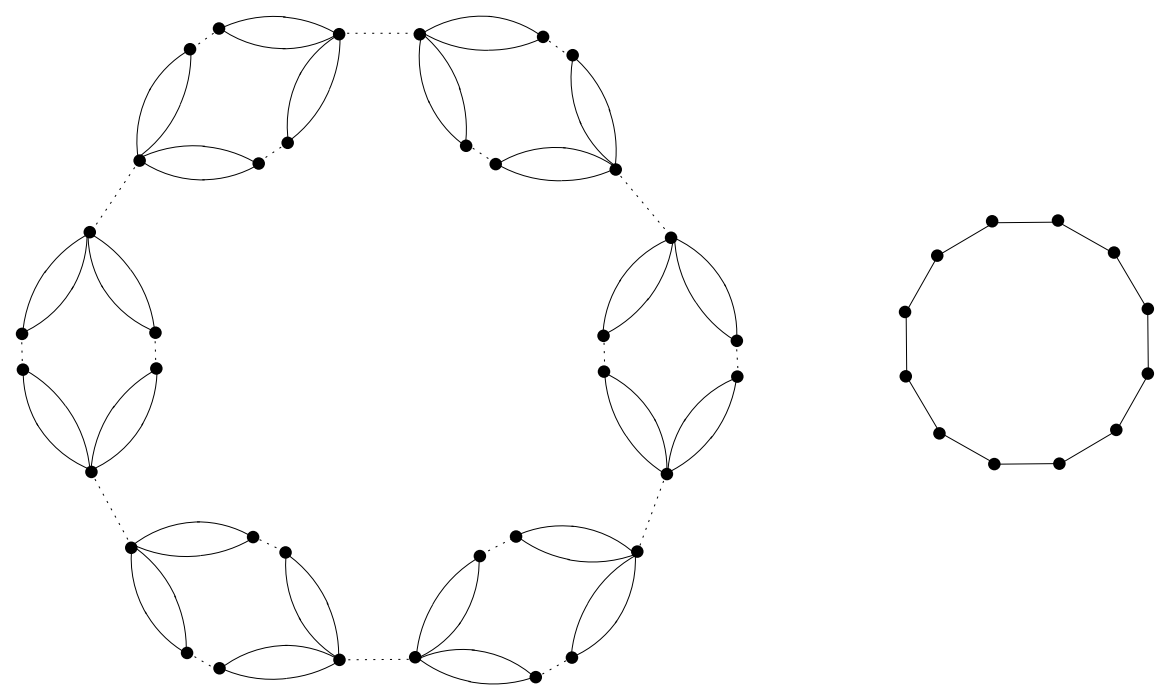

Figure 9: The graph $\mathrm{BC}\left(D_{6}^{\prime \prime}\right)$ (left) with each dotted line matching the two 'halves' of a splitted vertex of $D_{6}^{\prime \prime}$, and the graph $G_{D_{6}^{\prime \prime}}=C_{12}$ (right).

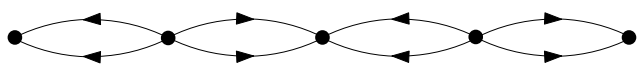

Figure 10: The open 4-necklace.

matching in a given graph covers as many vertices as possible. The maximum matching problem is the problem of finding a maximum matching in a given graph $G$. The number of edges in such a matching is called the matching number of $G$ and denoted $\alpha^{\prime}(G)$. Thanks to the pioneering work of Tutte and Edmonds, the maximum matching problem is known to be solvable in polynomial time. In particular, one of the 1965 papers of Edmonds on polyhedral combinatorics, describes, among other things, the so-called Blossom Algorithm [7] (see also [2], pp. 452)), an $O\left(n^{2} m\right)$ algorithm that finds a maximum matching in any given graph of order $n$ and size $m$. Over the years, various improvements of the Blossom Algorithm have been found (see, e.g., [14], pp. 422-423).

Our next result establishes a relationship between the defect $\operatorname{def}(D)$ of any given digraph $D$ and the order $n\left(G_{D}\right)$ and matching number $\alpha^{\prime}\left(G_{D}\right)$ of the corresponding simple graph $G_{D}$.

Theorem 2.8. For every digraph $D, \operatorname{def}(D)=n\left(G_{D}\right)-\alpha^{\prime}\left(G_{D}\right)$ holds.

Proof of Theorem 2.8. By Theorems 2.1 and 2.2, we may assume that $\chi_{\text {wo }}^{\prime}(D)=3$. Take an arbitrary edge coloring $\varphi$ of $D$ with color set $\{1,2\}$. For simplicity, we use the same notation $\varphi$ to denote the inherited edge coloring of $\operatorname{PS}(D)$. Let $\operatorname{PS}(D)_{1}$ and $\operatorname{PS}(D)_{2}$ be the spanning subgraphs of $\operatorname{PS}(D)$ whose respective edge sets are the color classes $\varphi^{-1}(1)$ and $\varphi^{-1}(2)$. For every vertex $x \in V(\operatorname{PS}(D))$, we abbreviate $d_{\mathrm{PS}(D)_{1}}(x)$ to $d_{1}(x)$, and likewise $d_{\mathrm{PS}(D)_{2}}(x)$ to $d_{2}(x)$. Consider the partition $\{V(D) \cap V(\operatorname{PS}(D)), V(D) \backslash V(\operatorname{PS}(D))\}$ of $V(D)$, and observe the following:

- a vertex $u \in V(D) \cap V(\operatorname{PS}(D))$ is 'defective' if and only if both $d_{1}(u)$ and $d_{2}(u)$ 
are odd;

- a vertex $v \in V(D) \backslash V(\operatorname{PS}(D))$ is 'defective' if and only if some $v^{ \pm} \in\left\{v^{+}, v^{-}\right\}$is a nonisolated vertex of $\operatorname{PS}(D)$ such that both $d_{1}\left(v^{ \pm}\right)$and $d_{2}\left(v^{ \pm}\right)$are even (possibly zero); call every such $v^{ \pm}$a 'defective half-vertex' originating from $v$.

First we show that each bad component of $\operatorname{PS}(D)$ 'contains' at least one defective vertex.

Claim 2.8.1. Each component $K$ of $\mathrm{BC}(D)$ contains a defective vertex or a defective half-vertex.

Proof of Claim 2.8.1. Let $K_{1}=K \cap \operatorname{PS}(D)_{1}$ and $K_{2}=K \cap \operatorname{PS}(D)_{2}$. Since $K$ is an even graph, clearly $\operatorname{OddV}\left(K_{1}\right)=\operatorname{OddV}\left(K_{2}\right)$ and $\operatorname{Even} V\left(K_{1}\right)=\operatorname{Even} V\left(K_{2}\right)$. By the above observations, within $V(K)$, the defective vertices constitute the set $V_{1} \cap \operatorname{OddV}\left(K_{1}\right)$ and the defective half-vertices constitute the set $V_{2} \cap \operatorname{Even} V\left(K_{1}\right)$. In order to show that the union of these two sets is nonempty it suffices to note that

$$
\left(V_{1} \cap \operatorname{Odd} V\left(K_{1}\right)\right) \cup\left(V_{2} \cap \operatorname{Even} V\left(K_{1}\right)\right)=\left(V_{2} \cap V(K)\right) \oplus \operatorname{OddV}\left(K_{1}\right),
$$

the right-hand side being the symmetric difference of $V_{2} \cap V(K)$ and $O d d V\left(K_{1}\right)$. Now observe that $V_{2} \cap V(K)$ is odd-sized by the assumption that $K$ is bad. And, since $\operatorname{Od} d V\left(K_{1}\right)$ is even-sized by the handshake lemma, we conclude that $\left(V_{2} \cap V(K)\right) \oplus \operatorname{OddV}\left(K_{1}\right)$ is odd-sized. Thus, the union of (2.1) is indeed nonempty, i.e., $K$ contains a defective vertex or a defective half-vertex.

We shall establish the desired equality $\operatorname{def}(D)=n\left(G_{D}\right)-\alpha^{\prime}\left(G_{D}\right)$ by showing that each of the two opposed inequalities $\operatorname{def}(D) \geq n\left(G_{D}\right)-\alpha^{\prime}\left(G_{D}\right)$ and $\operatorname{def}(D) \leq n\left(G_{D}\right)-$ $\alpha^{\prime}\left(G_{D}\right)$ holds. In order to demonstrate the former inequality, we will need the following auxiliary result.

Claim 2.8.2. Let $G[X, Y]$ be a simple bipartite graph such that for each vertex $v \in X$ the degree $d_{G}(v)$ is at most 2 and for each vertex $w \in Y$ the degree $d_{G}(w)$ is positive. If $|X|=m$ and $|Y|=n$ then $G$ contains at least $n-m$ pairwise vertex-disjoint 2-paths whose interior vertices belong to $X$.

Proof of Claim 2.8.2. Let $p_{2}(G)$ be the maximum size of a set of pairwise vertex-disjoint 2-paths in $G$ with all interior vertices in $X$. We prove that $p_{2}(G) \geq n-m$ by induction on the number $x_{2}(G)$ of 2 -vertices ${ }^{2}$ contained in $X$. If $x_{2}(G)=0$ then every vertex $v \in X$ is of degree at most 1 . Since every vertex $w \in Y$ is of degree at least 1 , we have that

$$
n-m=\sum_{w \in Y} 1-\sum_{v \in X} 1 \leq \sum_{w \in Y} d_{G}(w)-\sum_{v \in X} d_{G}(v)=0=p_{2}(G) .
$$

Assuming $x_{2}(G) \geq 1$, select a 2-vertex $v_{0} \in X$. Define $X^{\prime}=X \backslash\left\{v_{0}\right\}$ and $Y^{\prime}=Y \backslash N_{G}\left(v_{0}\right)$, and let $m^{\prime}=\left|X^{\prime}\right|$ and $n^{\prime}=\left|Y^{\prime}\right|$; thus, $m^{\prime}=m-1$ and $n^{\prime}=n-2$. Note that the induced subgraph $G^{\prime}\left[X^{\prime}, Y^{\prime}\right]$ meets the degree conditions. Since $x_{2}\left(G^{\prime}\right)=x_{2}(G)-1$, the inductive hypothesis gives $n^{\prime}-m^{\prime} \leq p_{2}\left(G^{\prime}\right)$. Therefore, as clearly $p_{2}\left(G^{\prime}\right) \leq p_{2}(G)-1$, we deduce that

$$
n-m=\left(n^{\prime}-m^{\prime}\right)+1 \leq p_{2}\left(G^{\prime}\right)+1 \leq p_{2}(G),
$$

which completes the inductive argument.

\footnotetext{
${ }^{2}$ A vertex $v$ of a graph $G$ is said to be a $d$-vertex if $d_{G}(x)=d$.
} 
We are ready to show one of the two opposed inequalities stated above.

Claim 2.8.3. $\operatorname{def}(D) \geq n\left(G_{D}\right)-\alpha^{\prime}\left(G_{D}\right)$.

Proof of Claim 2.8.3. Returning to an arbitrary edge coloring $\varphi$ of $D$ with color set $\{1,2\}$, we construct a simple bipartite graph $G[X, Y]$ as follows. Let $X$ be the set of defective vertices in $D$ under $\varphi$. Let $Y$ be the set of components of $\operatorname{BC}(D)$. Join a defective vertex $v$ with a bad component $K$ if $K$ contains $v$ or contains a defective half-vertex originating from $v$. By Claim 1, the obtained graph $G[X, Y]$ meets the requirements of Claim 2 . Consequently, there are $n\left(G_{D}\right)-|X|$ pairwise disjoint 2-paths in $G[X, Y]$ whose interiors belong to $X$. However, this clearly gives a matching in $G_{D}$ of size $n\left(G_{D}\right)-|X|$; simply for every such 2-path $y_{1} x y_{2}$ from $G[X, Y]$ assign the edge $v_{y_{1}} v_{y_{2}}$ to the matching of $G_{D}$. We conclude that $\alpha^{\prime}\left(G_{D}\right) \geq n\left(G_{D}\right)-|X|$. Equivalently, $|X| \geq n\left(G_{D}\right)-\alpha^{\prime}\left(G_{D}\right)$. The arbitrariness of $\varphi$ yields the desired inequality.

In order to complete the proof of Theorem 2.8 we also need to prove the opposite inequality.

Claim 2.8.4. $\operatorname{def}(D) \leq n\left(G_{D}\right)-\alpha^{\prime}\left(G_{D}\right)$.

Proof of Claim 2.8.4. Consider a maximum matching $M=\left\{v_{K_{2 i-1}} v_{K_{2 i}}: 1 \leq i \leq k\right\}$ in $G_{D}$. Returning to $\operatorname{PS}(D)$, for each $i \in\{1, \ldots, k\}$ let $v_{i} \in V_{2}$ be a vertex such that $\left\{v_{i}^{+}, v_{i}^{-}\right\}$ intersects both $K_{2 i-1}$ and $K_{2 i}$. We color the edges of each nonempty component $K$ of $\operatorname{PS}(D)$ as described below. And for this, we define first an even-sized subset $T$ of $V(K)$ :

- If $K \in\left\{K_{1}, K_{2}, \ldots, K_{2 k}\right\}$ then define $T=\left(V(K) \cap V_{2}\right) \backslash\left\{v_{1}^{+}, v_{1}^{-}, \ldots, v_{k}^{+}, v_{k}^{-}\right\}$. The intersection $V(K) \cap V_{2}$ is odd-sized and contains precisely one of the vertices $v_{1}^{+}, v_{1}^{-}, \ldots, v_{k}^{+}, v_{k}^{-}$; hence, $T$ is even-sized.

- If $K$ is a component of $\mathrm{BC}(D)-\bigcup_{i=1}^{2 k} K_{i}$ then there exists $w_{K} \in V_{2}$ such that the intersection $\left\{w_{K}^{+}, w_{K}^{-}\right\} \cap V(K)$ is a singleton; moreover, the 'other half' of $\left\{w_{K}^{+}, w_{K}^{-}\right\}$ does not fall into another component of $\mathrm{BC}(D)-\cup_{i=1}^{2 k} K_{i}$, by the maximality of $M$. Define $T=\left(V(K) \cap V_{2}\right) \backslash\left\{w_{K}^{+}, w_{K}^{-}\right\}$. Again, $V(K) \cap V_{2}$ is odd-sized and only one of the vertices $w_{K}^{+}, w_{K}^{-}$falls inside $V(K) \cap V_{2}$; consequently, $T$ is even-sized.

- If $K$ is not a component of $\mathrm{BC}(D)$ then (as in the proof of Theorem 2.2) we distinguish between two options: in case $V(K) \cap V_{2}$ is even-sized, define $T=V(K) \cap V_{2}$; otherwise, as $V(K) \cap V_{3} \neq \varnothing$, select an odd-sized subset $S \subseteq V(K) \cap V_{3}$ and define $T=\left(V(K) \cap V_{2}\right) \cup S$. Obviously, $T$ is even-sized.

By construction, $T$ is always an even-sized subset of $V(K)$. Therefore, there exists a $T$-join $H$ of $K$. Color $E(H)$ with 1 and $E(K) \backslash E(H)$ with 2. After this has been done for every component $K$ of $\operatorname{PS}(D)$, consider the inherited edge coloring of $D$. The set of its defective vertices is precisely $R=\left\{v_{1}, \ldots, v_{k}\right\} \cup\left\{w_{K}: K\right.$ is a component of $\left.\mathrm{BC}(D)-\cup_{i=1}^{2 k} K_{i}\right\}$. Indeed, by construction we have the following:

- no vertex $u \in V(D) \cap V(\operatorname{PS}(D))$ is defective because one of the colors 1 and 2 has an odd number of appearances on each of the odd-sized semi-cuts of $u$ (as both $d_{1}(u)$ and $d_{2}(u)$ are even); 
- a vertex $v \in V(D) \backslash V(\operatorname{PS}(D))$ is defective if and only if $v \in R$ because those are the only $v$ 's for which some $v^{ \pm} \in\left\{v^{+}, v^{-}\right\}$is a nonisolated vertex of $\operatorname{PS}(D)$ such that both $d_{1}\left(v^{ \pm}\right)$and $d_{2}\left(v^{ \pm}\right)$are even (possibly zero).

Thus, the total number of defective vertices equals $n\left(G_{D}\right)-\alpha^{\prime}\left(G_{D}\right)$, which confirms the desired inequality $\operatorname{def}(D) \leq n\left(G_{D}\right)-\alpha^{\prime}\left(G_{D}\right)$.

Proof of Theorem 2.8, continued: From Claims 2.8.3 and 2.8.4 it follows that $\operatorname{def}(D)=$ $n\left(G_{D}\right)-\alpha^{\prime}\left(G_{D}\right)$.

Let us reconsider our examples. Since $G_{D_{n}^{\prime}}=K_{1, n}$ and $G_{D_{n}^{\prime \prime}}=C_{2 n}$, we have $n\left(G_{D_{n}^{\prime}}\right)=$ $n+1$ and $\alpha^{\prime}\left(G_{D_{n}^{\prime}}\right)=1$, whereas $n\left(G_{D_{n}^{\prime \prime}}\right)=2 n$ and $\alpha^{\prime}\left(G_{D_{n}^{\prime \prime}}\right)^{n}=n$; thus, in view of Theo$\operatorname{rem} 2.8, \operatorname{def}\left(D_{n}^{\prime}\right)^{n}=\operatorname{def}\left(D_{n}^{\prime \prime}\right)=n$.

Note that Theorem 2.8 and Proposition 2.7 combined provide an answer to our previous question about the complexity of finding the defect of a digraph.

Proposition 2.9. The parameter $\operatorname{def}(D)$ can be determined in polynomial time. Moreover, the problem of finding the defect of a digraph is polynomially equivalent to the problem finding the matching number of a graph.

Another immediate consequence of Theorem 2.8 is the following.

Corollary 2.10. For every digraph $D$ it holds that

$$
\left\lceil\frac{n\left(G_{D}\right)}{2}\right\rceil \leq \operatorname{def}(D) \leq n\left(G_{D}\right) .
$$

With all being said, it is clear that, in general, there is no 'directed analogue' of Proposition 2.5, which served as our initial motivation here. In other words, there are digraphs that have arbitrarily many 'defective vertices'.

\section{Characterizations in terms of $\chi_{\mathrm{wo}}^{\prime}$ and def}

We consider two classes of digraphs: the class $\mathcal{A D}=\{D(G): G$ is a pseudograph $\}$ of digraphs $D(G)$ that are associated to graphs $G$, and the class $\mathcal{T}$ of tournaments.

\subsection{Associated digraphs}

We shall use here an additional convention hinted in the introduction. Namely, define $\chi_{\text {wo }}^{\prime}(G)=\infty$ for each graph $G$ that contains 'isolated loops'. The following theorem characterizes the associated digraphs in terms of their weak-odd chromatic index.

Theorem 3.1. For any connected graph $G$, it holds that

$$
\chi_{\mathrm{wo}}^{\prime}(D(G))= \begin{cases}0 & \text { if } G=K_{1}, \\ 1 & \text { if } G \text { is an odd graph }, \\ 3 & \text { if } G \text { is an even bipartite graph of odd order } \geq 3, \\ 2 & \text { otherwise. }\end{cases}
$$


. Let $D=D(G)$. It always holds that

$$
\chi_{\mathrm{wo}}^{\prime}(D) \leq \chi_{\mathrm{wo}}^{\prime}(G) .
$$

Indeed, say $\varphi$ is a weak-odd $\chi_{\text {wo }}^{\prime}(G)$-edge coloring of $G$. The accompanying edge coloring $\varphi_{D}$ of $D$ assigns to any pair of arcs stemming from an edge $e$ in $G$ color $\varphi(e)$. Consequently, $\varphi_{D}$ is a weak-odd $\chi_{\text {wo }}^{\prime}(G)$-edge coloring of $D(G)$, which settles (3.1).

Thus, the nontrivial part of Theorem 3.1 amounts to showing the following equivalence:

$$
\chi_{\mathrm{wo}}^{\prime}(D)=3 \Leftrightarrow \quad \Leftrightarrow \text { is an even bipartite graph of odd order } n(G) \geq 3 \text {. }
$$

In view of inequality (3.1) and Theorem 1.1, we may confine to $G$ being an even graph of odd order $n(G) \geq 3$. With that assumption, clearly $\operatorname{PS}(D)=\operatorname{BG}(D)$ and $V_{1}=V_{3}=\varnothing$. Therefore, by Theorem 2.2, the equality $\chi_{\mathrm{wo}}^{\prime}(D)=3$ holds true if and only if some nonempty component of $\mathrm{BG}(D)$ is of odd order. Consequently, the proof of equivalence (3.2) will be complete if we establish the following two assertions.

Claim 3.1.1. If $G$ bipartite, then $\mathrm{BG}(D)=G \bullet G$, i.e., $\mathrm{BG}(D)$ consists of two vertexdisjoint copies of $G$.

Claim 3.1.2. If $G$ is not bipartite, then $\mathrm{BG}(D)$ is connected.

A moment's reflection reveals that Claim 3.1.1 is implied by the definitions of 'associated digraph' and 'split'. For if $G=G\left[V_{1}, V_{2}\right]$ is a bipartite graph with bipartition $\left(V_{1}, V_{2}\right)$, then $\mathrm{BG}(D)=G\left[V_{1}^{+}, V_{2}^{-}\right] \cup G\left[V_{1}^{-}, V_{2}^{+}\right]$, that is, $\mathrm{BG}(D)$ is the disjoint union of two bipartite graphs, with respective bipartitions $\left(V_{1}^{+}, V_{2}^{-}\right)$and $\left(V_{1}^{-}, V_{2}^{+}\right)$, each of which is isomorphic to $G$.

As for the demonstration of Claim 3.1.2, let $x, y$ be an arbitrary pair of (not necessarily distinct) vertices of $G$ (and thus of $D$ ). In order to show the existence of an $x^{+}-y^{-}$walk in $\operatorname{BG}(D)$, it suffices to find an $x-y$ walk of odd length in $G$. Indeed, any such walk $W: x v_{1} v_{2} \cdots v_{2 k} y$ would yield a walk $W^{ \pm}: x^{+} v_{1}^{-} v_{2}^{+} \cdots v_{2 k-1}^{-} v_{2 k}^{+} y^{-}$in $\mathrm{BG}(D)$.

Consider an odd cycle $C$ of $G$. Let $P$ and $Q$, respectively, be an $x-C$ and a $y-C$ path in $G$. Denote by $v_{x}$ and $v_{y}$ the (not necessarily distinct) endpoints of $P$ and $Q$ in $C$. Of the two $v_{x} v_{y}$ arcs of $C$, let $L$ be the one whose length is of opposite parity than the combined length $\ell_{P}+\ell_{Q}$ of $P$ and $Q$. Then $P \cup L \cup Q$ gives rise to a desired $x-y$ walk of odd length.

A similar argument proves the existence of an $x^{+}-y^{+}$walk in $\mathrm{BG}(D)$; it suffices to find an $x-y$ walk of even length in $G$, which can be done by using the other $v_{x} v_{y}$ arc of $C$ in the previous argument. The existence of $x^{-}-y^{+}$and $x^{-}-y^{-}$walks in $\operatorname{BG}(D)$ for an arbitrary pair of vertices $x$ and $y$ in $G$ now follows by symmetry.

An immediate consequence of Theorem 3.1 and inequality (3.1) is the following.

Corollary 3.2. If $G$ is a connected graph, then $\chi_{\mathrm{wo}}^{\prime}(D(G)) \leq \chi_{\mathrm{wo}}^{\prime}(G)$. Moreover, equality holds unless $G$ is an even nonbipartite graph of odd order.

Let us characterize the associated digraphs in terms of their defect.

Proposition 3.3. For any connected graph $G$, it holds that

$$
\operatorname{def}(D(G))= \begin{cases}1 & \text { if } G \text { is an even bipartite graph of odd order } \geq 3 \\ 0 & \text { otherwise. }\end{cases}
$$


Proof. By Theorem 3.1, unless $G$ is an even bipartite graph of odd order $n(G) \geq 3$, it holds that $\operatorname{def}(D(G))=0$. On the other hand, assuming $G$ is an even bipartite graph of odd order $n(G) \geq 3$, by the proof of Theorem 3.1, $\mathrm{PS}(D)=\mathrm{BG}(D)=G \cup G$. Thus, $\mathrm{BC}(D)=G \cup G$ and $G_{D(G)}=K_{2}$. Consequently, by Theorem 2.8, $\operatorname{def}\left(D_{G}\right)=1$.

Taking into account the established inequality $\operatorname{def}(D(G)) \leq 1$, one naturally wonders if an analogue of Proposition 2.5 holds for all associated digraphs. The following proposition answers this in the positive.

Proposition 3.4. Every connected associated digraph $D$ admits a 2-edge coloring such that condition $(\overrightarrow{\mathrm{WO}})$ is satisfied at each vertex apart from a prescribed vertex $v \in V(D)$.

Proof. Let $D=D(G)$. We may assume that $G$ is an even bipartite graph of odd order $n(G) \geq 3$. As already observed in the proof of Proposition 3.3, it holds that $\operatorname{PS}(D)=G \cup G$. Let $T=V(G) \backslash\{v\}$, and take a $T$-join $H$ of $G$. Color the edges of $\operatorname{PS}(D)$ with color set $\{1,2\}$ as follows: in each copy of $G$, color $E(H)$ by 1 and $E(G) \backslash E(H)$ by 2. The inherited 2-edge coloring of $D$ meets the requirements.

\subsection{Tournaments}

In view of Proposition 2.4, there exist tournaments that require three colors for a weak-odd edge coloring; namely, as every tournament of odd order with a single peripheral vertex meets the requirements of the aforementioned proposition, its weak-odd chromatic index equals 3. Our characterization below asserts that those tournaments are the only 'exceptions' to weak-odd 2-edge colorability in the class $\mathcal{T}$. The proof shall make use of the following classical results on tournaments.

Given a digraph $D$, spanning directed paths and cycles are referred to as hamiltonian paths and hamiltonian cycles, respectively. Back in 1959, Camion [3] proved that a nontrivial tournament is strong if and only if it contains a hamiltonian cycle. (In fact, this basic result was later on improved, first by Harary and Moser [8], and shortly after by Moon, see, e.g., [9], but for our purposes the initial result of Camion will suffice.) Another basic theorem on tournaments of an even earlier date, due to Rédei [13], is that every tournament (not necessarily strong) has a hamiltonian path. (In fact, Rédei [13] proved that every tournament contains an odd number of hamiltonian paths.)

Theorem 3.5. For any tournament $T$, it holds that

$$
\chi_{\mathrm{wo}}^{\prime}(T)= \begin{cases}0 & \text { if } T=K_{1}, \\ 1 & \text { if } T \text { is nontrivial and every vertex semi-degree is odd or zero, } \\ 3 & \text { if } T \text { is nontrivial, of odd order, and has just one peripheral vertex, } \\ 2 & \text { otherwise. }\end{cases}
$$

Proof. For simplicity of presentation, call every nontrivial tournament of odd order having only one peripheral vertex bad and call every other tournament good. By Proposition 2.4 and Theorem 2.1, the nontrivial aspect of the proof consists of showing that:

Every good tournament is weak-odd 2-edge colorable.

Consider a good tournament $T$. If it has two peripheral vertices, then the following furnishes a weak-odd 2-edge coloring: take a hamiltonian path $P$ of $T$, color $A(P)$ with 
1 and the rest of $A(T)$ with 2 . Since the initial (resp. terminal) vertex of $P$ is the source (resp. sink) of $T$, color 1 satisfies condition $(\overrightarrow{\mathrm{WO}})$ at all vertices of $T$. Similarly, if every strong component of $T$ is nontrivial, then a simple construction of a weak-odd 2-edge coloring can be obtained as follows: in every strong component $K$ of $T$ take a hamiltonian cycle $C_{K}$, color $\cup_{K} A\left(C_{K}\right)$ with 1 and the rest of $A(T)$ with 2 . Then, color 1 meets condition $(\overrightarrow{\mathrm{WO}})$ everywhere.

Hence, we may assume that there exist a nontrivial peripheral strong component and a trivial strong component of $T$. We complete the proof by distinguishing between two cases.

Case 1: Both peripheral strong components of $T$ are nontrivial. Let $K_{i}$ and $K_{t}$ be the initial and terminal strong components of $T$. There exists a directed $K_{i}-K_{t}$ path $P$ in $T$ that passes through every vertex $v \notin V\left(K_{i}\right) \cup V\left(K_{j}\right)$. Indeed, simply take a hamiltonian path in the 'multitournament' $T /\left\{K_{i}, K_{t}\right\}$, i.e., the directed multigraph obtained from $T$ by contracting $V\left(K_{i}\right)$ and $V\left(K_{t}\right)$ into a pair of new vertices. By the above assumptions, the path $P$ is of length $\ell(P)>1$. Denote by $x$ and $y$, respectively, the initial and terminal vertex of $P$. Thus, the arc $x y \in A(T) \backslash A(P)$. Let $C_{i}$ and $C_{t}$, respectively, be hamiltonian cycles in $K_{i}$ and $K_{j}$. The arc set $A\left(C_{i}\right) \cup A(P) \cup\{x y\} \cup A\left(C_{t}\right)$ induces a spanning subdigraph of $T$ with all semi-degrees odd. By coloring $A\left(C_{i}\right) \cup A(P) \cup\{x y\} \cup A\left(C_{t}\right)$ with 1 and the rest of $A(T)$ with 2 we complete a weak-odd 2-edge coloring of $T$, because color 1 meets condition $(\overrightarrow{\mathrm{WO}})$ everywhere.

Case 2: One peripheral strong component of $T$ is trivial. Since $T$ is good, it has even order. We may assume $T$ has a sink, say $y$. Let $K_{i}$ be the initial strong component of $T$, and $C_{i}$ be a hamiltonian cycle in $K_{i}$. If $V(T)=V\left(K_{i}\right) \cup\{y\}$, then we are done by coloring $A\left(C_{i}\right)$ with 1 and the rest of $A(T)$ with 2 . Namely, color 2 meets condition ( $\overrightarrow{\mathrm{WO}}$ ) at $y$, and color 1 takes care of every other vertex.

Assuming $V(T) \neq V\left(K_{i}\right) \cup\{y\}$, take a directed $K_{i}$-y path $P$ in $T$ that passes through every vertex $v \notin V\left(K_{i}\right)$ (a hamiltonian path in the 'multitournament' $T / K_{i}$, the directed multigraph obtained from $T$ by contracting $V\left(K_{i}\right)$ into a vertex, will do). Let $x$ be the initial vertex of $P$; thus, $V\left(C_{i}\right) \cap V(P)=\{x\}$. By our latest assumption, the arc $x y \notin$ $A(P)$. Consequently, the arc set $A\left(C_{i}\right) \cup A(P) \cup\{x y\}$ induces a spanning subdigraph of $T$ such that both the semi-degrees of $y$ are even whereas the rest of the semi-degrees are odd. Therefore, as $d_{T}(y)$ is odd, by coloring $A\left(C_{i}\right) \cup A(P) \cup\{x y\}$ with 1 and the rest of $A(T)$ with 2 we obtain a weak-odd 2 -edge coloring of $T$. Indeed, once again color 2 meets condition $(\overrightarrow{\mathrm{WO}})$ at $y$, and color 1 takes care of every other vertex.

Let us characterize the members of the class $\mathcal{T}$ in terms of their defect.

Proposition 3.6. For any tournament $T$, it holds that

$$
\operatorname{def}(T)= \begin{cases}1 & \text { if } T \text { is nontrivial, of odd order, and just one vertex semi-degree is zero, } \\ 0 & \text { otherwise. }\end{cases}
$$

Proof. By Theorem 3.5, we may assume that $T$ is nontrivial, of odd order, and just one vertex semi-degree is zero. Say $T$ has a sink $y$. Apply to $E(T)$ the particular 2-edge coloring(s) constructed for Case 2 in the proof of Theorem 3.5. Observe that condition $(\overrightarrow{\mathrm{WO}})$ is satisfied at each vertex apart from $y$. 
Therefore, as for the class of associated digraphs, the inequality $\operatorname{def}(T) \leq 1$ holds for every tournament $T$. Our final proposition shows that an analogue of Proposition 2.5 also holds for all tournaments.

Proposition 3.7. Every tournament $T$ admits a 2-edge coloring such that condition $(\overrightarrow{\mathrm{WO}})$ is satisfied at each vertex apart from a prescribed vertex $v \in V(T)$.

Proof. Again, we may assume that $T$ is nontrivial, of odd order, and with just one peripheral vertex, say a sink $y$. Note that BG(T) has only two components, and moreover, one of those components consists of the isolated vertex $y^{+}$. Indeed, by our assumptions, every vertex $w \in V(T) \backslash\{y\}$ dominates $y$ and has $d_{T}^{-}(w)>0$; hence, the component containing $y^{-}$ also includes both $w^{+}$and $w^{-}$. Consequently, $\operatorname{PS}(T)$ has only one nonempty component $K$ and only one empty component $\left\{y^{+}\right\}$. Observe that $V(K) \cap V_{2}=V_{2} \backslash\left\{y^{+}\right\}$is odd-sized, and $V_{3}=\varnothing$. Define an even-sized subset $S \subseteq V(K)$ as follows:

- if $v \in V_{1}$ then $S=\{v\} \cup\left(V_{2} \backslash\left\{y^{+}\right\}\right)$;

- if $v \notin V_{1}$ then $S=V_{2} \backslash\left\{v^{-}, y^{+}\right\}$.

The rest should be clear. We simply take an $S$-join $H$ of $K$, and then color $E(H)$ with 1 and $E(K) \backslash E(H)$ with 2 . The inherited 2-edge coloring of $T$ meets the requirements.

\section{Concluding remarks and further work}

For a graph $G$ (resp. digraph $D$ ), an edge covering with color set $S$ is a mapping that assigns to each edge of $G$ (resp. arc of $D$ ) a nonempty subset of $S$; what distinguishes coverings from colorings is that we allow more than one color per edge (resp. arc). Related notions to weak-odd edge colorings of graphs and digraphs, respectively, are the weak-odd edge coverings defined as edge coverings such that conditions (WO) and $(\overrightarrow{\mathrm{WO}})$ are fulfilled. It is known that most of the graphs and digraphs are weak-odd 3-edge colorable. Can a color always be saved by switching to coverings? The answer to this question in the realm of graphs is affirmative. Indeed, the following holds true.

Proposition 4.1. Any connected graph $G$ whose edge set does not consist entirely of loops, admits a weak-odd 2-edge covering such that the intersection of color classes is contained within a prescribed singleton $\{e\} \subseteq E(G)$.

Proof. By Theorem 1.1, we may assume that $G$ is a nontrivial even graph of odd order. Subdivide the edge $e$, and take an odd factor $H$ of the obtained graph. Color $E(G) \cap E(H)$ with $1, E(G) \backslash(E(H) \cup\{e\})$ with 2 , and assign both colors 1 and 2 to the edge $e$. It is readily seen that the constructed edge covering meets the requirements.

Following this line of reasoning, we find the next question interesting.

Question 4.2. Does every digraph admit a weak-odd 2-edge covering?

Presuming Question 4.2 answers in positive, define ovl $(D)$, the overlapping of $D$, to be the minimum possible size of the intersection of the two color classes in an arbitrary weak-odd 2-edge covering of $D$. In view of the families of digraphs $D_{n}^{\prime}$ and $D_{n}^{\prime \prime}$ (depicted in Figure 7), it is easily seen that ovl $(D)$ is not bounded over the class of digraphs; moreover, it can acquire any possible value from the set of naturals. We are tempted to 
wonder whether this parameter also relates to some 'classical graph parameter', much as like $\operatorname{def}(D)$ relates to the maximum matching number of graphs.

Following the direction explored in Section 3, it may be interesting to characterize other digraph families in terms of their weak-odd chromatic index and their defect. Since tournaments proved to have a nice behavior with respect these parameters, a natural next step is to consider families of digraphs generalizing tournaments.

Three classic generalizations of tournaments that come to mind are semicomplete digraphs, extended tournaments and multipartite tournaments. A digraph is semicomplete if it is obtained from a complete graph by replacing each edge $u v$ by the arc $(u, v)$, the arc $(v, u)$ or the pair of arcs $(u, v)$ and $(v, u)$. An extended tournament is a digraph obtained from a tournament by blowing up some of its vertices into stable sets. A multipartite tournament is an orientation of a complete multipartite graph.

Problem 4.3. Characterize the families of semicomplete digraphs, extended tournaments and multipartite tournaments in terms of their weak-odd chromatic index.

We think that the following question should be addressed before stating the analogous problem for the characterization in terms of the defect.

Question 4.4. Is there a constant $c$ such that $\operatorname{def}(D) \leq c$ for every digraph $D$ such that

- $D$ is semicomplete?

- $D$ is an extended tournament?

- $D$ is a multipartite tournament?

A positive answer for Question 4.4 would open the door to consider the following problem.

Problem 4.5. Characterize the families of semicomplete digraphs, extended tournaments and multipartite tournaments in terms of their defect.

\section{ORCID iDs}

César Hernández-Cruz (D) https://orcid.org/0000-0002-5867-3801

Mirko Petruševski (D) https://orcid.org/0000-0001-8048-7418

Riste Škrekovski (D) https://orcid.org/0000-0001-6851-3214

\section{References}

[1] J. Bang-Jensen and G. Z. Gutin, Digraphs. Theory, Algorithms and Applications, Springer, London, 2nd edition, 2009, doi:10.1007/978-1-84800-998-1.

[2] J. A. Bondy and U. S. R. Murty, Graph Theory, Springer, New York, 2008, https : / / i ink. springer.com/book/9781846289699.

[3] P. Camion, Chemins et circuits hamiltoniens des graphes complets, C. R. Acad. Sci., Paris 249 (1959), 2151-2152.

[4] P. Cheilaris, B. Keszegh and D. Pálvölgyi, Unique-maximum and conflict-free coloring for hypergraphs and tree graphs, SIAM Journal on Discrete Mathematics 27 (2013), 1775-1787, doi: $10.1137 / 120880471$. 
[5] J. Czap and S. Jendrol', Colouring vertices of plane graphs under restrictions given by faces, Discuss. Math. - Graph Theory 29 (2009), 521-543, doi:10.7151/dmgt.1462.

[6] J. Czap, S. Jendrol', F. Kardoš and R. Soták, Facial parity edge colouring of plane pseudographs, Discrete Math. 312 (2012), 2735-2740, doi:10.1016/j.disc.2012.03.036.

[7] J. Edmonds, Paths, trees, and flowers, Can. J. Math. 17 (1965), 449-467, doi:10.4153/ CJM-1965-045-4.

[8] F. Harary and L. Moser, The theory of round robin tournaments, Am. Math. Mon. 73 (1966), 231-246, doi:10.2307/2315334.

[9] J. W. Moon, Topics on Tournaments in Graph Theory, Dover Publications, Inc., Mineola, New York, 2015.

[10] M. Petruševski, A note on weak odd edge-colorings of graphs, Adv. Math. Sci. J. 4 (2015), 7-10, https://www.researchgate.net/publication/297020049_A_ NOTE_ON_WEAK_ODD_EDGE-COLORINGS_OF_GRAPHS.

[11] M. Petruševski and R. Škrekovski, Weak-odd edge-coloring of digraphs, Mat. Bilt. 37 (2013), 61-74, doi:10.37560/matbil13100061p.

[12] L. Pyber, Covering the edges of a graph by..., in: Sets, Graphs and Numbers, Colloquia Mathematica Societatis János Bolyai, volume 60, pp. 583-610, 1991, https: / / korandi.org/ setsgraphsnumbers.html.

[13] L. Rédei, Ein kombinatorischer Satz, Acta Litt. Sci. Szeged 7 (1934), 39-43, http: / / pub. acta.hu/acta/customer/showVolume.ftl.

[14] A. Schrijver, Combinatorial optimization. Polyhedra and efficiency (3 volumes), volume 24 of Algorithms Comb., Springer, Berlin, 2003, https://link.springer.com/book/ 9783540443896. 